\title{
Nurse's impressions and changes after the workshops using the pediatric nursing care model
}

\author{
Naomi Matsumori * \\ Department of Nursing, Faculty of Health and Welfare, Prefectural University of Hiroshima, Hiroshima, Japan
}

Received: February 23, 2016

DOI: $10.5430 /$ jnep.v6n9p82
Accepted: April 20, 2016

URL: http://dx.doi.org/10.5430/jnep.v6n9p82

\begin{abstract}
For children undergoing medical examinations/procedures and nursing care, medical settings are unfamiliar environments where they are surrounded by unfamiliar people, so they often experience more fears and anxieties than adults would expect. To reduce them, nurses practice various types of patient care related to preparation, and pay adequate attention to providing psychological preparation when involved with children and their families. The workshops were conducted with the aims of: interpreting the meaning of the responses of children and their families, reflecting on daily nursing practice, exchanging information on nursing practice provided at other hospitals, and helping participants achieve new insights, by using the pediatric nursing care model. To hold workshops using a pediatric nursing care model demonstrating the basic attitudes towards ethical practice of nursing, including psychological preparation provided in medical settings, and investigate nurses' impressions of and changes observed after the workshops. 12 pediatric nurses working at medical institutions in Japan were participated with informed consent. As a result of this workshops using the pediatric nursing care model, the original goals were accomplished. Furthermore, the implementation rate has improved in most of the care model items after the third workshop, showing the positive outcome of the workshops. We would like to further refine the model, and hold more workshops by improving its content and methods.
\end{abstract}

Key Words: Pediatric nursing, Care model, Nurse's impressions and changes, Workshop

\section{INTRODUCTION}

\subsection{Introduce the problem}

For children undergoing medical examinations/procedures and nursing care, medical settings are unfamiliar environments where they are surrounded by unfamiliar people, so they often experience more fears and anxieties than adults would expect. To reduce them, nurses practice various types of patient care related to preparation, and pay adequate attention to providing psychological preparation when involved with children and their families. We developed a pediatric nursing care model summarizing basic attitudes toward the ethical practice of nursing, including preparation, based on the data of nursing practice examples obtained through in- terviews and participant observation in actual medical settings. ${ }^{[1]}$ The model indicates important points to consider in the practice of nursing, particularly in medical care settings, while protecting the dignity of children and parents. This can be applied to the practice of pediatric nursing if the basic ideas and perspectives of each item can be well understood.

We held workshops, and investigated participants' impressions of and changes observed after the workshops, with the aims of assessing their practice while focusing on the meaning of the behavior of children undergoing medical examinations/procedures and their parents, sharing the practice of each participant, and exchanging information among

\footnotetext{
*Correspondence: Naomi Matsumori; Email: matumori@pu-hiroshima.ac.jp; Address: Department of Nursing, Faculty of Health and Welfare, Prefectural University of Hiroshima, 723-0053 1-1, Gakuencho, Mihara, Hiroshima, Japan.
} 
participants, by using the pediatric nursing care model developed by us.

\subsection{Background}

The need for preparation to reduce psychological aggression among children undergoing medical care has been recognized since the 1930s, and it was introduced into the field of pediatric nursing around 1970 in Europe and the United States. Nursing practices for children reflected the psychological influence of meeting the parents, the disease, and hospitalization. Between 1940 and 1960 preparation programs using a drawing method, a pre-hospital tour, pamphlets or picture books, and puppets were de-veloped. ${ }^{[2]}$

In Japan, a large number of articles and translations regarding psychological responses of children to hospitalization and illness as well as their countermeasures have been published since around the 1970s, and preoperative orientation and discharge instructions for children and their parents were initiated. In 1990, with the publication of a report on informed consent by the Japan Medical Association, patients' rights to know started to be respected and became common in medical fields, and adherence to "Children's right to be informed in a manner appropriate to age and understanding", Article 13 of the "Convention on the Rights of the Child" which was ratified by Japan in 1994, has been discussed in the context of medical practice. ${ }^{[3]}$ The Committee on Bioethics of the American Academy of Pediatrics (AAP) published a policy statement titled, "Informed consent, parental permission, and assent in pediatric practice" in 1995. The AAP defined assent as having the following elements: "Making a clinical assessment of the patient's understanding of the situation and the factors influencing how he or she is responding, helping the patient achieve an awareness of his or her condition for him or her to willingly accept the proposed care, and telling the patient what he or she can expect with tests and treatment(s)", by which participation of children in decision-making and their involvement in discussions about their health care were advocated to best serve the concept of assent. ${ }^{[4]}$

Nurses' awareness of ethics in nursing practice has steadily improved in the pediatric setting through the increased implementation of and surveys on the care to protect the dignity of children and their parents, such as psychological preparation. However, Komiya et al., in their study which investigated ethical awareness of 30 pediatric nurses, reported that $40 \%$ of the subjects responded that they can understand and practice "respect for the right to know and make decisions", but it was mostly practiced towards parents and rarely toward children. ${ }^{[5]}$ Takahashi et al. investigated the current status of the protection of children's rights in nursing settings involving 295 nurses working in university or pediatric hospitals, and reported that the implementation of measures to resolve cases of children's rights violation remained at the discussion level among nurses, and the ethical practice of nursing has not been implemented among nurses with less than 5 years of pediatric nursing experience. Among various types of education, lifelong learning had the greatest impact on ethical attitudes of nurses, suggesting the importance of providing education to working nurses to resolve the lack of ethical practice in nursing. ${ }^{[6]}$

Our survey suggests that although awareness of psychological preparation for children undergoing medical procedures in Japan has improved among nurses, it seems that the current status regarding its provision has not. Furthermore, a large majority of nursing staff working on mixed wards need to learn more specialized knowledge regarding child care, especially in terms of preparation. The actual meaning of preparation also needs to be clarified among nurses for whom it remains ambiguous. In 1994, resulting from the ratification of the Convention of the Rights of the Child in Japan, numerous books and journals introduced psychological preparation as a method to protect the rights of children in Japanese medical settings. This suggests that both the psychological and ethical meanings of preparation need to be considered. To promote the application of psychological preparation, seminars and workshops regarding the improvement of nurse's cognition are needed. ${ }^{[7]}$

\subsection{Describe relevant scholarship}

McInally reports on Child Health Nurses' (CHNs') perceptions of the impact of pediatric oncology education on their practice. Findings suggest that formal pediatric oncology nurse education is perceived to positively impact on certain aspects of the practitioners practice. All the CHNs felt empowered through their newly found knowledge, confidence and attitude, and ability to apply evidence-based care. All participants perceived a need for education at all levels, most enjoyed the blended approach to teaching, learning, and assessment, however all experienced great support from one another and the sharing of personal and professional experiences. ${ }^{[8]}$ Marta reports the findings about the approach to evaluate the impact of training and a Best Practice Guide on nurses' knowledge and their perceptions of the application of family-centered care, a new concept in pediatric nurses' daily practice being at the early stages in Portugal. The sample consisted of 24 nurses in a pediatric unit, and they answered three questionnaires. Positive differences were confirmed between nurses' knowledge about family-centered care and their perceptions in relation to the application of this philosophy in their daily practice before and after the training, and implementation of the Best Practice Guide. The 
nursing care model represents important possibilities to develop the knowledge of nursing. ${ }^{[9]}$ Rocha et al. evaluated the Nursing Care Model for Children Victimized of Violence. The model was elaborated utilizing the instrument for evaluation of nursing care models, and conducted by a panel of 18 specialists. The analysis includes analysis of objective responses and other texts prepared by experts as part of its assessment process. The results show that the process of Evaluation of the Care Models is essential that they are evaluated to promote the understanding of those who analyze them, and a willingness is needed to keep the momentum of implementation. They stated that evaluation and reformulation are continuous process, and if they are perfected and meet the physical/material world, as well as being spiritual and sensible, then they are present in every action of nursing care. ${ }^{[10]}$ Hunsberger reports the effect of introducing parents of hospitalized children to the nursing mutual participation model of care (NMPMC). If Nurses are introduced to the NMPMC, and parents are given the NMPMC information, parents will experience less anxiety at discharge, and have a higher level of comfort in mutual participation activities. Nurses themselves rate at a higher level of performance in mutual participation behavior than parents who have not been introduced to the NMPMC. ${ }^{[11]}$ Curtis et al. report how models of care applying family-centered care principles targeting critically ill children and their families can create positive changes in care delivery for the family. A model which provides continuity across the span of care is required. They suggest that there is a need to describe how best to design, implement and sustain models of care for critically ill children and their families. The success of any intervention implementation will be dependent on the comprehensiveness of the strategy for implementation, the relevance to the context and setting, and engagement with key stakeholders. ${ }^{[12]}$

\subsection{Study purpose}

To hold workshops using a pediatric nursing care model demonstrating the basic attitudes towards ethical practice of nursing, including psychological preparation provided in medical settings, and investigate nurses' impressions of and changes observed after the workshops.

\section{METHOD}

\subsection{Participants and ethical considerations}

Twelve pediatric nurses working at medical institutions in Japan were participated. A letter of invitation outlining the research aims and providing further details of the study accompanied each questionnaire. Participants were informed that their anonymity would be protected and that their participation was voluntary. Questionnaires did not contain any personal information that could identify the respondents.

\subsection{Design}

A 2-hour workshop was held three times. At the first workshop, the general outline of a pediatric nursing care model was explained and its practice examples were introduced to the participants. At the second workshop, participants were asked to describe their practice on a written form. At the third workshop, the written examples of their practice were then presented and shared among the participants. The second workshop was held two weeks after the first workshop, and the third workshop was held one month after the second workshop.

The implementation rate of 24 items of the pediatric nursing care model (PNCM cheklist), and impressions of the workshops were investigated during the first and third workshops for observation of nurses' changes.

\subsection{Data collection and analysis}

PNCM cheklist, which were developed based on the original version (2006) by Matsumori et al., ${ }^{[1]}$ as the reflection of nursing practice by each nurses and the data collection tool, were investigated during the workshops and the results of the first and third workshops were compared. PNCM cheklist of each item, which was rated using a 4-point Likert scale ("always" to "never"), was calculated using Microsoft Excel (Microsoft Corporation, Santa Rosa, CA). Concerning an implementation rating scale, preliminary tests $(n=39)$ were conducted to assess item consistency (Cronbach's $\alpha=.973$ ).

Twenty-four items of PNCM cheklist: (1) You greet children and introduce yourself to them to inform them that you are the nurse in charge. (2) One of the physicians, nurses, or parents provides children with explanations of medical examinations/procedures or confirms them in advance. (3) You ask children in advance when they want to be informed of medical examinations/procedures. (4) You inform children of when medical examinations/procedures are implemented. (5) When deciding whether or not parents should accompany their children (at the time of providing explanations/conducting medical examinations and procedures), you take into consideration the requests of children and their parents. (6) You ensure that both parents and children know where the parents will wait. (7) You also inform parents of the explanations and content of the methods provided for children. (8) You explain medical examinations/procedures (including their purposes and methods) to children, using easy-to-understand expressions, even when their parents are present. (9) If children resist, you wait patiently until they change their minds. (10) You do the best you can to prevent children from being fearful. (11) You provide children 
with explanations and talk to them in each stage of medical examinations/procedures. (12) You appropriately respond to children's questions and remarks. (13) When children cry, you implement other appropriate measures rather than forcing them to obey. (14) You allow children to bring in their favorite things. (15) You distract children's attention away from medical examinations/procedures. (16) When it is taking longer than expected to conduct medical examinations/procedures, you inform parents of their progress. (17) You avoid chatting with other health care professionals about subjects not related to medical examinations/procedures. (18) When examinations/procedures have not yet been completed, you avoid using expressions that may lead children and their parents to mistakenly think that they have been completed. (19) You verbally inform children and their parents that examinations/procedures have been completed. (20) You praise children for having been brave. (21) You consider the feelings of parents, saying: "You must have been worried". (22) You encourage parents to praise their children for having been brave. (23) Following the completion of medical examinations/procedures, you provide children with instructions to be followed. (24) You check the responses of children following the implementation of medical examinations/procedures.

The responses to the open-ended questions received from the nurses were categorized based on content, and are presented in brackets in this report. Within each category, some representative responses were quoted directly.

\section{RESULTS}

A total of 12 nurses participated in the first workshop, but was reduced to 9 at the second workshop due to work-related reasons. Since all answers were kept anonymous, corresponding samples could not be identified. Therefore, responses obtained in the first and third workshops were tabulated by dividing them into the following phases: (1) Prior to, (2) during, and (3) following the implementation of medical examinations or procedures, and the ratio of the denominator was presented in a graph form to compare them.

\subsection{Changes in the results of the checklist in each phase \\ 3.1.1 Prior to the implementation of medical examina- tions and procedures (see Figure 1)}

A comparison of the percentage of participants who reported "Always" showed that the implementation rate has increased in all items.

The implementation rate of items: “(1) You greet children and introduce yourself to them to inform them that you are the nurse in charge", "(4) You inform children of when medical examinations/procedures are implemented”, “(7) You also inform patients of the explanations and content of the

Published by Sciedu Press methods provided for children”, “(8) You explain medical examinations/procedures to children, using easy-to-understand expressions", and "(9) If children resist, you wait patiently until they change their minds", was $50 \%, 33 \%, 0,25 \%$, and $25 \%$ in the first workshop, but increased to $67 \%, 56 \%, 33 \%$, $78 \%$, and $56 \%$ in the third workshop, respectively.

\subsubsection{During the implementation of medical examinations and procedures (see Figure 2)}

A comparison of the percentage of participants who responded "Always" showed that the implementation rate has increased in 6 of the 8 items, e.g., the implementation rate of items: "(11) You provide children with explanations and talk to them in each stage of medical examinations/procedures", "(14) You allow children to bring in their favorite things", and "(15) You distract children's attention away from medical examinations/procedures", was 33,58 , and $25 \%$ in the first workshop, but increased to $56 \%, 78 \%$, and $56 \%$ in the third workshop, respectively.

\subsubsection{Following the implementation of medical examina- tions and procedures (see Figure 3)}

A comparison of the percentage of participants who responded "Always" showed that the implementation rate has increased in 2 of the 6 items. However, the implementation rate of items: "(19) You verbally inform children and their parents that examinations/procedures have been completed" and "(20) You praise children for having been brave", remained the same $(100 \%$ in both the first and third workshops). That of the item, "(24) You check the responses of children following the implementation of medical examinations/procedures", was reduced from $50 \%$ to $33 \%$ after the third workshop, suggesting the need to emphasize this as a future issue.

\subsection{Participants' impressions regarding the first and third workshops (see Table 1)}

Participants' impressions regarding each workshop were summarized as follows:

\subsubsection{Participants' impressions regarding the first work- shop}

[Changes in the perceptions of psychological preparation] were observed among the participants, as they stated that "provision of explanations using tools is not the only methods of psychological preparation", and they also recognized the importance of reflecting on "the entire process of explanations and involvements".

They also stated that "I became aware of what I can do and have to work on" and "I realized that I rarely checked the responses of children following the implementation of examinations", indicating that they have achieved [insights into 
their practice] through the identification of what they have done and need to do. [The practice provided in other hospitals served as useful information] in the participants, but at the same time, they developed [concerns regarding their practice], such as "I have no idea how long I have to wait until children change their minds", by reflecting on their current practices.

Also, they stated that "I would like to use the model as a tool to reflect on my daily nursing practice" and "I would like to make opportunities to share ideas among other nurses", indicating that the model has contributed to motivating them [Translating reflection into practice].

Table 1. Participants' impressions regarding the first and third workshops (excerpts)

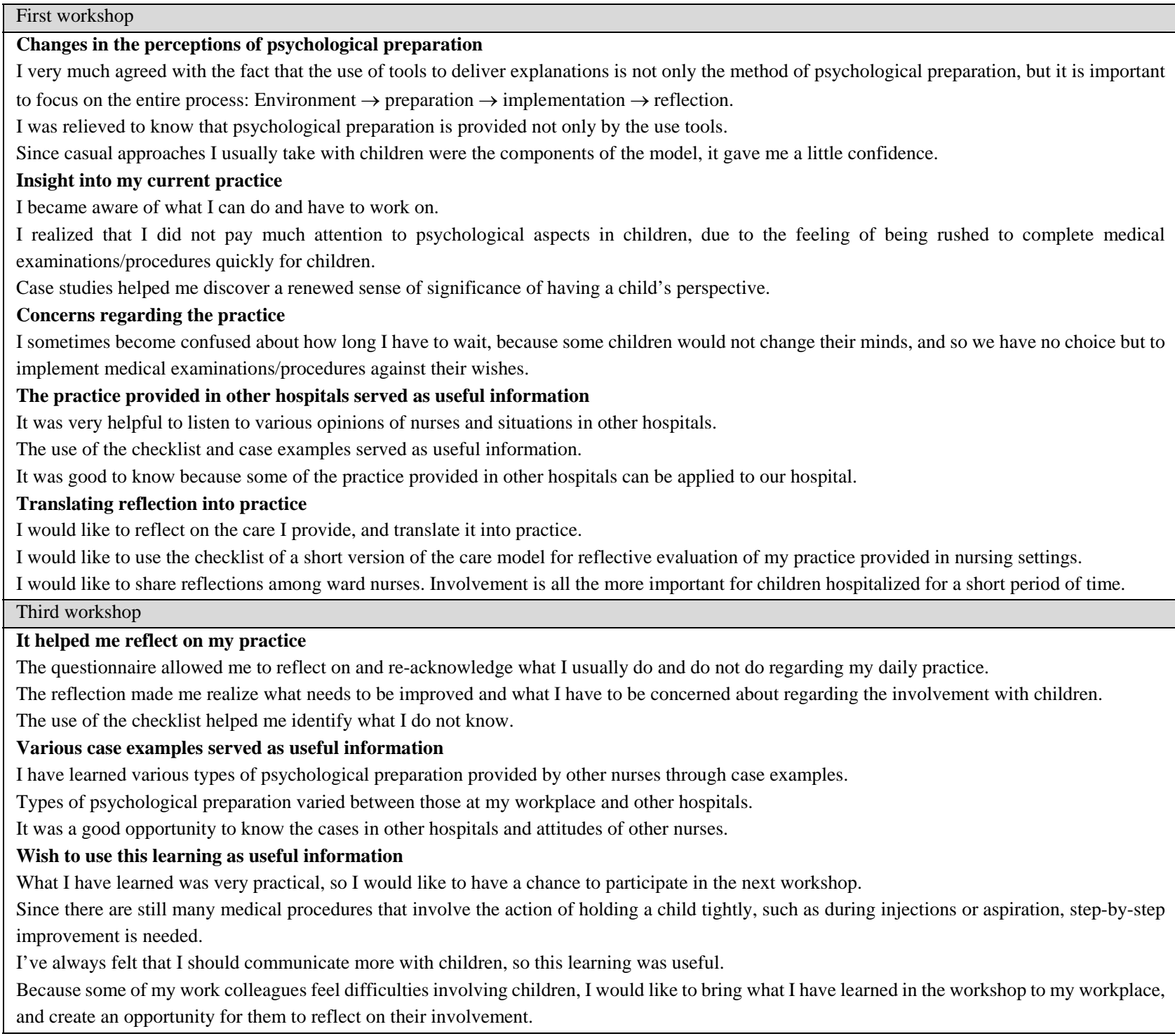

\subsubsection{Participants' impressions regarding the third work-} shop

The participants stated the outcomes of the workshops, such as "The questionnaire allowed me to reflect on and reacknowledge what I usually do and do not do regarding my daily practice", showing that the workshops helped them [reflect on their practice], and "I have learned various types of psychological preparation provided by other nurses through case examples", indicating that [various case ex86 amples served as useful information]. As another outcome, they also stated that "Because some of my colleagues feel difficulties involving children, I would like to bring what I have learned in the workshop to my workplace, and create an opportunity for them to reflect on their involvement", and showed a [wish to use this learning as useful information] not only for the reflection on their practice, but also for instructions of their hospital staff members. 


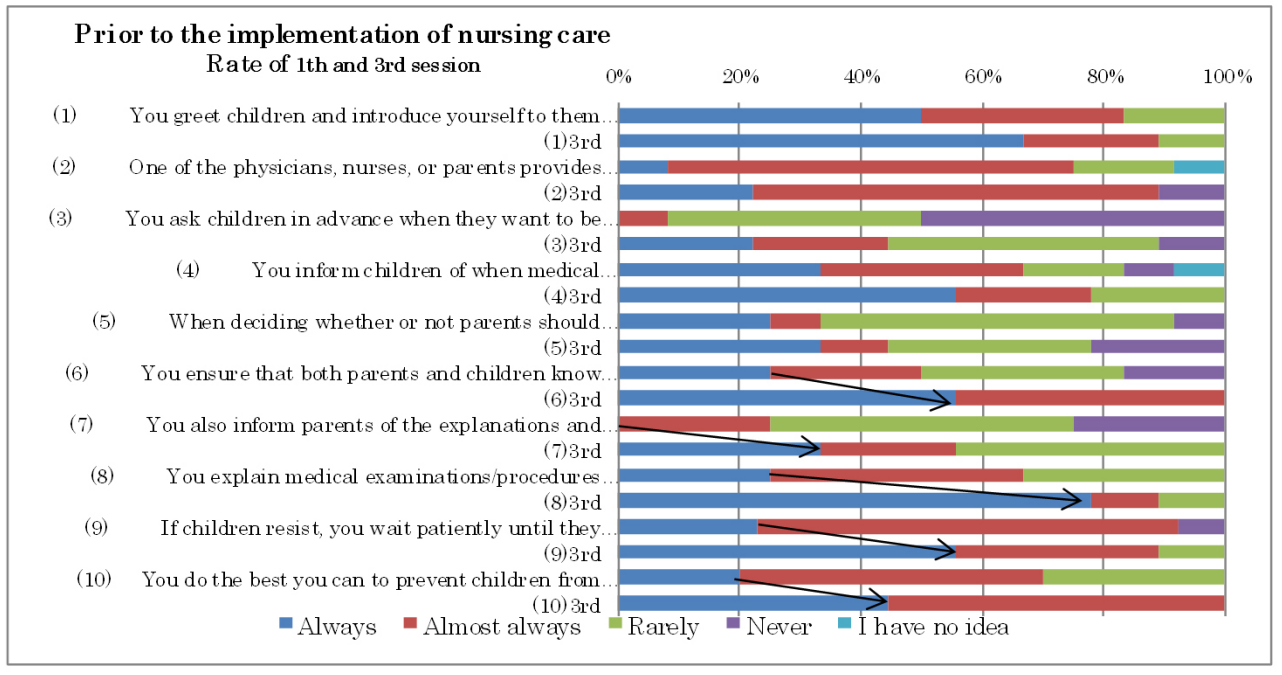

Figure 1. Change of rate of Prior to the implementation of nursing care

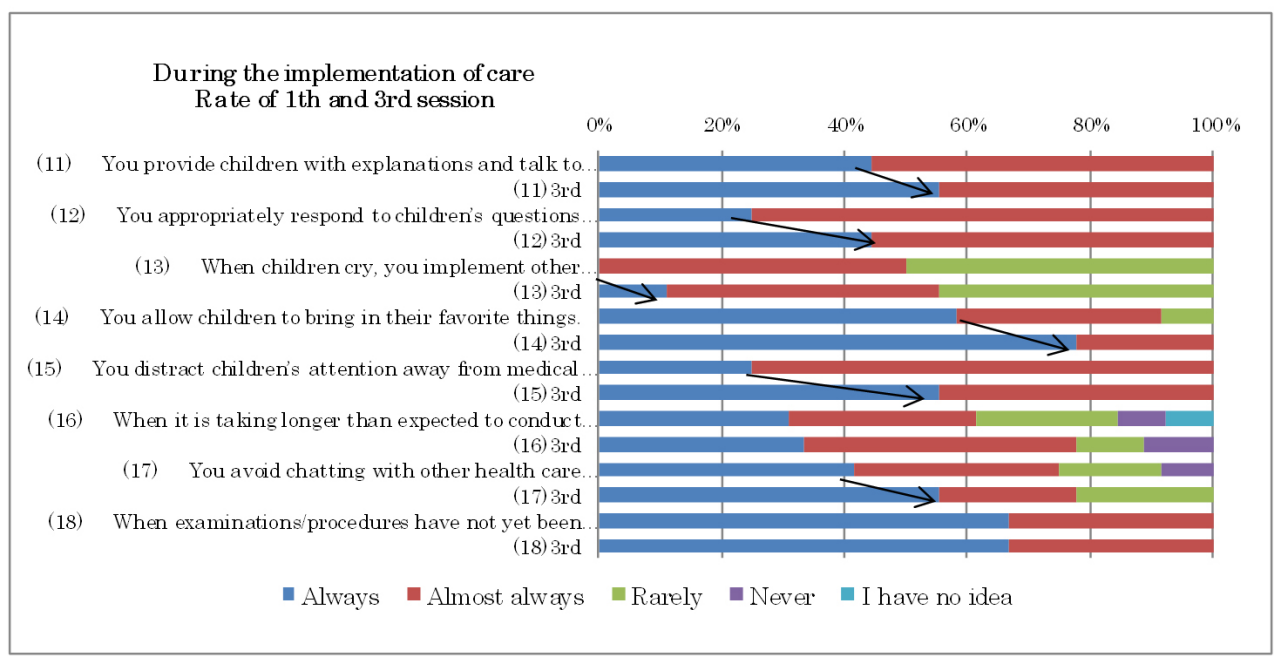

Figure 2. Change of rate of During the implementation of nursing care

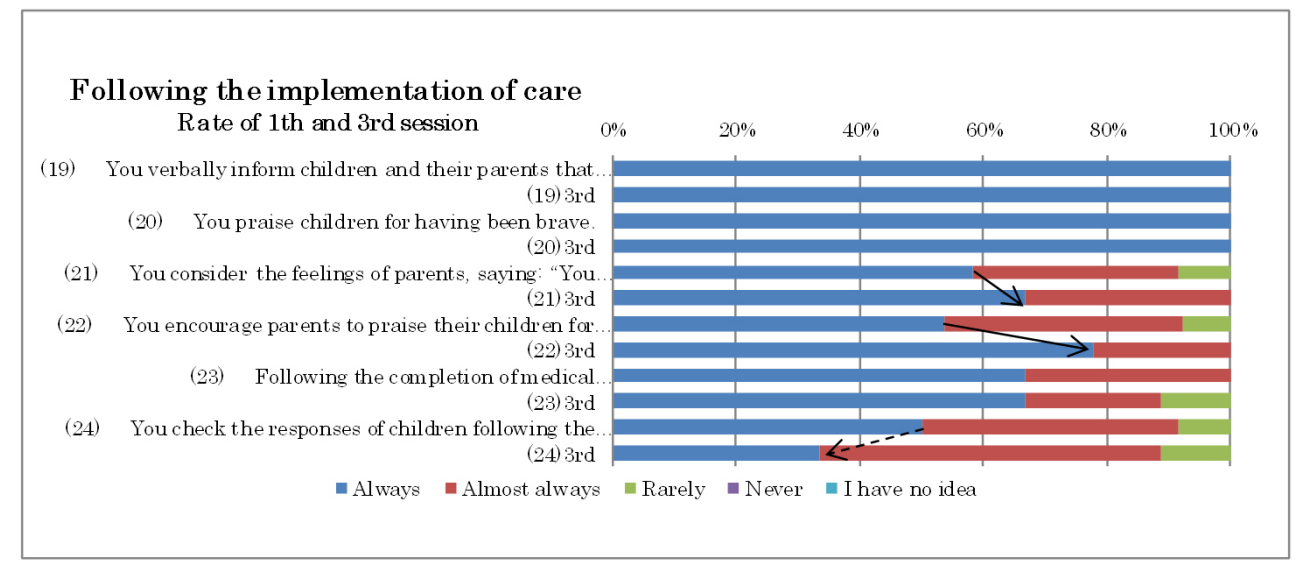

Figure 3. Change of rate of Following the implementation of nursing care 


\section{Discussion}

In many cases, it is difficult to put nursing care models into practice only by the reading or understanding of their items. We, therefore, conducted workshops based on the results of the study in which the original version of the care model was introduced to clinical nurses, and they achieved new findings and a practical sense. In the workshops, we explained the purpose and interpretation of each case example, and asked the participants to reflect on and verbalize their daily practice. As a result, the implementation rate of all (1) to (10) pre-treatment support items increased in the first and third workshops, possibly because they made intentional efforts to verbalize their daily practices, such as "waiting until children change their minds", "providing children with information they require", and "offering children a choice", in order to perform them in clinical settings. Also, sharing examples of nursing practice of other participants may have provided them with opportunities to understand problems of and the methods of practice performed by other nurses who are also involved with children and their families, and to make best use of them in their daily practice. Through the process of reflective evaluation using the pediatric nursing care model, even the participants who considered that their practice was inappropriate could also realize that they have actually practiced child-centered care. During the workshops, the participants could exchange their opinions while mutually appreciating each other's practice and efforts. In this workshops, some participants stated that the practice of other nurses serves as useful information for considering the methods of involvement with pediatric patients. They were able to not only share information, but also developed confidence through mutual appreciation of each other's practice, and had an opportunity to discuss better involvement with patients using examples of their practice.

As a result of this workshops using the pediatric nursing care model, the participants could achieve [changes in their perceptions of psychological preparation] and [new discoveries and insights into their care]. Also, they were motivated by the care model as shown in their comments, such as: [the practice provided in other hospitals served as useful information],
[It helped me to reflect on my practice], [I would like to translate reflection into practice], and [I wish to use the model as useful information], indicating that the original goals were accomplished. Furthermore, the implementation rate has improved in most of the care model items after the third workshop, showing the positive outcome of the workshops. We would like to refine the model depending on the comprehensiveness of the strategy for implementation. Therefore we have to hold more workshops by improving its content and methods. Further studies should be conducted with an increased sample size to facilitate practical application of the pediatric nursing care model.

\section{Conclusions}

The workshops were conducted with the aims of: interpreting the meaning of the responses of children and their families, reflecting on daily nursing practice, exchanging information on nursing practice provided at other hospitals, and helping participants achieve new insights, by using the pediatric nursing care model. As a result of this workshops using the pediatric nursing care model, the original goals were accomplished. Furthermore, the implementation rate has improved in most of the care model items after the third workshop, showing the positive outcome of the workshops. The results of the study are not generalizable because we can never know whether the case we have investigated is representative of the wider instances and the longer length of efficacy at the workshop. This research would provide insight for further research, then it needs to increase the sample size and the longer term investigation. We would like to further refine the model, and hold more workshops by improving its content and methods.

\section{ACKNOWLEDGEMENTS}

I would like to thank all the participants who assisted with data collection. This study was supported by Grants-in-Aid for Scientific Research in Japan 2010 and 2015.

\section{CONFLICTS OF INTEREST Disclosure}

The authors declare that there is no conflict of interest.

\section{REFERENCES}

[1] Mastumori N, Ninomiya K, Ebina M, et al. Practical application and evaluation of a care model for informing and reassuring children undergoing medical examinations and/or procedures (part 2): Methods of relating and practical nursing techniques that best bring out the potential of children. Japan Journal of Nursing Science. 2006; 3: 51-64. http://dx.doi.org/10.1111/j.1742-7924.2006.00052.x

[2] Bar-Mor G. Preparation of Children for Surgery and Invasive Pro- cedures: Milestones on the Way to Success. Journal of Pediatric Nursing. 1997; 12(4): 252-255. http://dx.doi.org/10.1016/S $0882-5963$ (97) $80010-3$

[3] Oikawa I. Preparation; ways and devices, Why is preparation required? Japanese Journal of Child Nursing. 2002; 25(2): 189-192 (in Japanese)

[4] American Academy of Pediatrics. Informed consent, parental permission, and assent in pediatric practice. Committee on Bioethics, 
American Academy of Pediatrics. Pediatrics. 1995; 95(2): 314-317. PMid:7838658.

[5] Komiya A, Asami Y, Fukuchi M, et al. The state of the ethical practice at pediatric nursing- the survey of cognition of nurses-(Japanese). 36th Japanese nursing society memoir pediatric nursing. 2006: 339341.

[6] Takahashi K, Hamanaka K, Arakawa M. Interrelation between Advocacy of Children's Rights and Experience of Ethics Education in Nursing (Japanese). The Japanese Journal of Health Science Research. 2014; 18(1): 21-31.

[7] Matsumori N. Psychological Preparation of Children for Medical Procedures: An Awareness Survey Targeting Nurses in Japan. Open Journal of Nursing. 2015; 5: 613-621. http://dx.doi.org/10. 4236/ojn.2015.57065

[8] McInally W, Masters H, Key S. The impact of paediatric oncology education on clinical practice - A phenomenological study. European
Journal of Oncology Nursing. 2012; 16(5): 498-504. PMid:22265663. http://dx.doi.org/10.1016/j.ejon.2011.12.001

[9] Marta ICG A. Family-centered care: impact of training and a best practice guide in pediatrics (Spanish). Revista de Enfermagem Referência (REFERENCIA). 2012; 7: 83-92.

[10] RochaI PK, Prad ML, Conroy S, et al. Evaluation of the nursing care model for children victimized of violence. Texto Contexto Enferm, Florianópolis. 2012; 21(1): 52-8. http://dx.doi.org/10.1590 /S0104-07072012000100006

[11] Hunsberger $M$. The effect of introducing parents of hospitalized children to the nursing mutual participation model of care: A randomized controlled trial. University of Waterloo Doctor of philosophy in Health Studies, Ontario, CANADA, 2000.

[12] Curtis K, Foster K, Mitchell R, et al. Models of Care Delivery for Families of Critically Ill Children: An Integrative Review of International Literature. Journal of Pediatric Nursing. 2015. http: //dx.doi.org/10.1016/j.pedn.2015.11.009 